Review

\title{
Heat Shock Protein 90 in Plants: Molecular Mechanisms and Roles in Stress Responses
}

\author{
Zhao-Shi Xu, Zhi-Yong Li, Yang Chen, Ming Chen, Lian-Cheng Li and You-Zhi Ma * \\ Institute of Crop Science, Chinese Academy of Agricultural Sciences (CAAS)/National Key Facility \\ for Crop Gene Resources and Genetic Improvement, Key Laboratory of Biology and Genetic \\ Improvement of Triticeae Crops, Ministry of Agriculture, Beijing 100081, China; E-Mails: \\ xuzhaoshi@yahoo.com.cn (Z.-S.X.); wangwangsd2010@yahoo.com.cn (Z.-Y.L.); \\ chenyang712@126.com (Y.C.); chenming@mail.caas.net.cn (M.C.); lilch@mail.caas.net.cn (L.-C.L.) \\ * Author to whom correspondence should be addressed; E-Mail: mayouzhi@yahoo.com.cn; \\ Tel.: +86-10-6891-9718; Fax: +86-10-6891-8789.
}

Received: 28 September 2012; in revised form: 29 October 2012 / Accepted: 29 October 2012 / Published: 23 November 2012

\begin{abstract}
The heat shock protein 90 (Hsp90) family mediates stress signal transduction, and plays important roles in the control of normal growth of human cells and in promoting development of tumor cells. Hsp90s have become a currently important subject in cellular immunity, signal transduction, and anti-cancer research. Studies on the physiological functions of Hsp90s began much later in plants than in animals and fungi. Significant progress has been made in understanding complex mechanisms of HSP90s in plants, including ATPase-coupled conformational changes and interactions with cochaperone proteins. A wide range of signaling proteins interact with HSP90s. Recent studies revealed that plant Hsp90s are important in plant development, environmental stress response, and disease and pest resistance. In this study, the plant HSP90 family was classified into three clusters on the basis of phylogenetic relationships, gene structure, and biological functions. We discuss the molecular functions of Hsp90s, and systematically review recent progress of Hsp90 research in plants.
\end{abstract}

Keywords: plant Hsp90; interaction mechanism; disease resistance; abiotic stress tolerance; phylogenetic relationship 


\section{Introduction}

Heat shock proteins (Hsps) are widely distributed in fungi, animals, and plants. Hsp transcripts significantly upregulate under high-temperature stress, and play an important role in heat stress response in living cells [1]. Hsps are mainly located in the cytoplasm under normal physiological conditions, but rapidly transfer to the nucleus under stress conditions. In accordance with molecular weight, Hsps are divided into several families, such as Hsp110, Hsp90, Hsp70/Hsp80, Hsp60, and small molecular Hsp (smHsp). The smHsp family were initially proposed to serve as molecular chaperones [2,3]. Now it has been demonstrated that Hsp90, Hsp70, and Hsp60 also function as molecular chaperones. Since the role of Arabidopsis Hsp90 in stress responses is known from the early studies, recent studies have showed that Hsp90 might play an important role in biological stress responses.

Hsp90s, highly conserved in molecular evolution, are involved in regulating and maintaining conformation of a variety of proteins, and in assisting normal cell survival under stresses [4]. In fungi and animals, Hsp90s mediate extensive stress signal transduction, including a role in folding of steroid hormone receptors, protein kinases, and transcription factors, as well as activation of the substrate to initiate stress signal transduction [5-8]. Recent studies showed that Hsp90s play an important role in controlling normal growth of human cell and in promoting tumor cell development [8,9]. Many oncoproteins are targets of Hsp90s, and Hsp90 inhibition can result in multipathway anti-tumor effects [10,11]. Inhibition of Hsp90 activity contributes to degradation of oncoproteins, helping in cancer treatment $[5,8,12,13]$. Cellular functions of the molecular chaperone activities of Hsp90s have been intensively studied in fungi and mammalian model systems. In contrast, studies on the physiological functions of Hsp90s in plants are more recent. A number of HSP90 genes have been identified from many plants, and they were strongly induced by changes in temperature, salinity, and heavy metals [14-18]. Molecular mechanisms of plant Hsp90s in response to stress, their roles as molecular chaperones, and their functions in enhancing plant resistance are not clear. It was recently shown that Hsp90s play an important role in plant development, stress response, and disease resistance [19-21].

Hsp90s directly influence canalization, assimilation, and rapid evolutionary alterations of phenotype through concealment and exposure of cryptic genetic variation [22]. Therefore, in-depth investigation of plant Hsp90 functions may help in understanding stress signal transduction, discovering pivotal stress-related genes, and improving the crop resistance. This paper reviews recent research on the molecular mechanisms of plant Hsp90s in order to promote their study, especially in regard to their manipulation in achieving biotic and abiotic stress resistance in crop plants.

\section{Structural and Functional Analyses of Hsp90}

Hsp90s are abundant (i.e., $\sim 1 \%$ of total proteins) in prokaryotic and eukaryotic cells. They are essential for viability in eukaryotes. Hsp90 increases to as much as $4 \%-6 \%$ of total protein under heat stress conditions [6,23]. They usually maintain a homodimer form that greatly increases in an emergency. They are mainly present in cytoplasm, and only rarely appear in the endoplasmic reticulum, mitochondria, and chloroplasts [24,25]. Hsp90s in the cytoplasm of animals is divided into

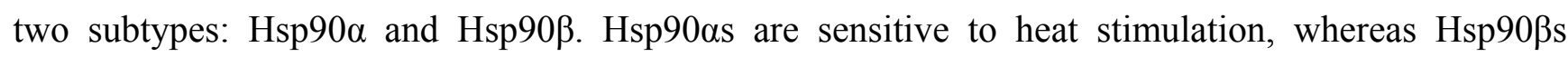
respond to mitotic induction. Hsp90as mainly contribute to protein transportation, protein folding, and 
maintenance of normal conformation preventing aggregation with ATP. Hsp90 $\beta$ is involved in the utilization of steroid hormones [26]. Hsp90s play different roles in prokaryotes and eukaryotes and are not necessary for the growth of prokaryotes [27,28].

Hsp90s serve as molecular chaperones in dimers regulated by ATP. Almost all of the Hsp90 family members are conserved, containing three domains (Figure 1): viz. an $N$ terminal conserved ATP-binding domain, a middle domain (M), and a $C$-terminal dimerization domain [23,29-31].

Figure 1. Structural model of Hsp90. Hsp90 consist of three domains: an $N$-terminal ATP-binding domain $(\mathrm{N})$; a middle domain $(\mathrm{M})$; and a $C$-terminal dimerization domain $(\mathrm{C})$ with the pentapeptide MEEVD sequence. A charged region exists between the $\mathrm{N}$ and $\mathrm{M}$ domains. All three domains are reported to interact with different substrates.

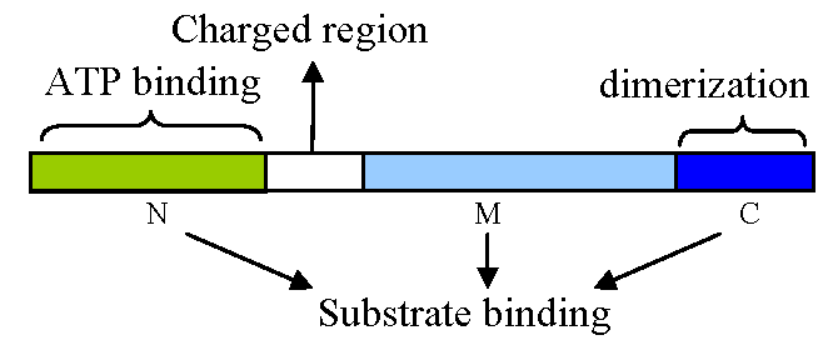

The ATP-binding domain of the Hsp90 N-terminal is the ATP/ADP binding site with intrinsic ATPase activity. Conformational changes in Hsp90s occur in the presence of ATP and then contribute to signal transduction, protein folding, and morphological evolution. ATP binding and hydrolysis cause conformational transitions of Hsp90 between an open conformation with the $\mathrm{N}$-domains separated and a closed conformation with the $N$-domains associated [32,33]. Mutation analysis showed that ATPase activity was necessary for the biological functions of Hsp90 [29,34]. Some natural substances, such as geldanamycin, closely combine with this position to interfere with Hsp90 functions, and then promote rapid degradation of protein kinase [35].

The middle domain of Hsp90 plays a key role in binding to substrate. Hsp90 and DNA topoisomerase II gyrase B (GyrB) share a similar structure, indicating the middle domain of Hsp90 as a primary substrate binding site. The middle domain contains a catalytic ring which can sense the presence of ATP- $\gamma$ phosphate and combine with it. The function of Hsp90 depends not only on the $N$-terminal domain, but also on the participation of the middle domain [36,37].

The $C$-terminal domain of Hsp90 is necessary for dimerization, and also functions as the binding site of calmodulin and other substrates. In eukaryotic cytoplasm, the $C$-terminal domain of Hsp90 contains a conserved five peptide domain (MEEVD) that is essential for the interaction between Hsp90 and proteins containing a tetratricopeptide repeat (TPR) domain. HSP70 also interacts with the TPR domain by an eight amino acid domain (GPTIEEVD) in a similar way to Hsp90 [5,29].

There is a charge zone consisting of about 50 amino acid residues between the $N$-terminal domain and the middle domain of Hsp90 in eukaryotes [38]. This area is not necessary for Hsp90, and mainly assists in covalent linkages, coordinating the $N$-terminal domain and the middle domain to maintain the conformation of Hsp90 binding with ATP. In fact, all three domains ( $N$-terminal, middle region and $C$-terminal) of Hsp90 were reported to be involved in binding with substrate proteins [29]. A general theory of interaction between Hsp90s and substrate proteins was formulated [8]: Hsp90s cannot form 
dimers without combining with ATP, and capture substrate proteins by two separate $N$-terminals. When the $N$-terminal of Hsp90 is combined with ATP, its conformation is changed to a ring formation to wrap the substrate protein. Hsp90 has to bind with the substrate to maintain a so-called "non-active state" (Figure 2).

Figure 2. The Hsp90 molecular clamp driven by ATP [8]. In the absence of ATP (A), Hsp90 is dimerized at its C-terminus in the "open" conformation. Upon ATP binding, the $N$-terminal domains undergo conformational changes that result in closing a "lid" over the bound nucleotide, and formation of a second dimerization interface between the amino-termini (B). Continued rearrangements of the closed conformation allow interaction of the N-terminal and middle domains resulting in the "closed and twisted" conformation which is able to hydrolyze ATP $(\mathbf{C})$.

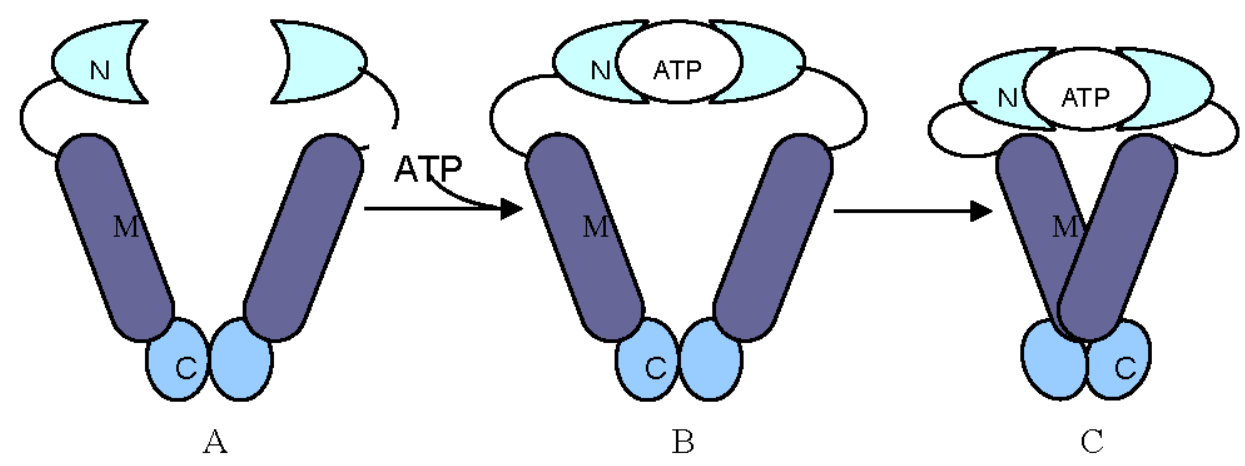

\section{Interactions of Proteins with Hsp90s and Their Interaction Mechanism}

Hsp90 participates in signal transduction indirectly by interaction with other proteins. Therefore, the loss of Hsp90 function may cause a variety of physiological defects in cells. More than 600 proteins interact with Hsp90 in yeast two-hybrid tests, tandem affinity chromatography, and genetic analyses in yeast [39]. Proteins interacting with Hsp90, mainly group into three types, include auxiliary proteins (co-chaperones), regulatory factors (regulators), and substrate proteins (substrates) (Table 1). Auxiliary proteins aid Hsp90 by binding with substrate proteins to maintain their stability. Many studies show that Hsp90 is regulated by phosphorylation, but the mechanism of regulation is still not clear. Eukaryotic Hsp90 proteins require accessory proteins and regulatory factors to regulate the interaction between themselves and substrate proteins, which together confer the diverse physiological activities of Hsp90 (Figure 3).

\subsection{Accessory Proteins}

Accessory proteins play a key role in regulating the ATP enzymatic activity of Hsp90s in cytoplasm and in mediating interactions between Hsp90s and substrates [8,40]. Most of the accessory proteins contain a TPR domain, and mainly depend on this domain to combine with the conservative $C$-terminal pentapeptide MEEVD of Hsp90. Each Hsp90 contains only one specific TPR-binding site. The TPR domain-containing proteins that interact with Hsp90 include HOP protein (corresponding to yeast Sti1), pro-free prime FKBP51 and FKBP52, cyclophilin Cyp40 (corresponding to yeast Cpr6 and 
Cpr7), protein phosphatase 5, yeast Cns1, Drosophila Dpit47, E3 ubiquitin ligase CHIP, and myosin-binding protein UNC-45. Although there are many TPR domain-containing proteins in plants, there are few reports besides ROF1 about their interaction with Hsp90 [41].

P23, a small acidic protein with chaperone activity, combines with the middle section of the $N$-terminal domain of Hsp90. It specifically identifies the binding state of Hsp90-ATP and plays a special role in the regulation of the Hsp90 ATPase cycle to promote the assembly and decomposition of the steroid receptor transcription complex [42]. Recent studies revealed that the activity of PIDD (p53-induced protein with a cell death domain) was regulated by a p23-Hsp90 complex [43] and PIDD mediated the activation of DNA repair by NF-kappaB [44]. The $C$-terminal of Cdc37 combines with the flanking region of Hsp90 at the $N$-terminal and may be the auxiliary protein of one specific substrate of Hsp90. Cdc37 regulates Hsp90 in specifically combining with protein kinase [45]. Recent research showed that Hsp90 allied with cyclophilin A to promote selective transportation of substrate, but the exact mechanism remains unclear [46]. These observations reveal that accessory proteins competitively binding to Hsp90 or substrate proteins generate different physiological functions.

Figure 3. Functions of Hsp90 and co-chaperones identified by interaction with substrates in different developmental phases in cells.

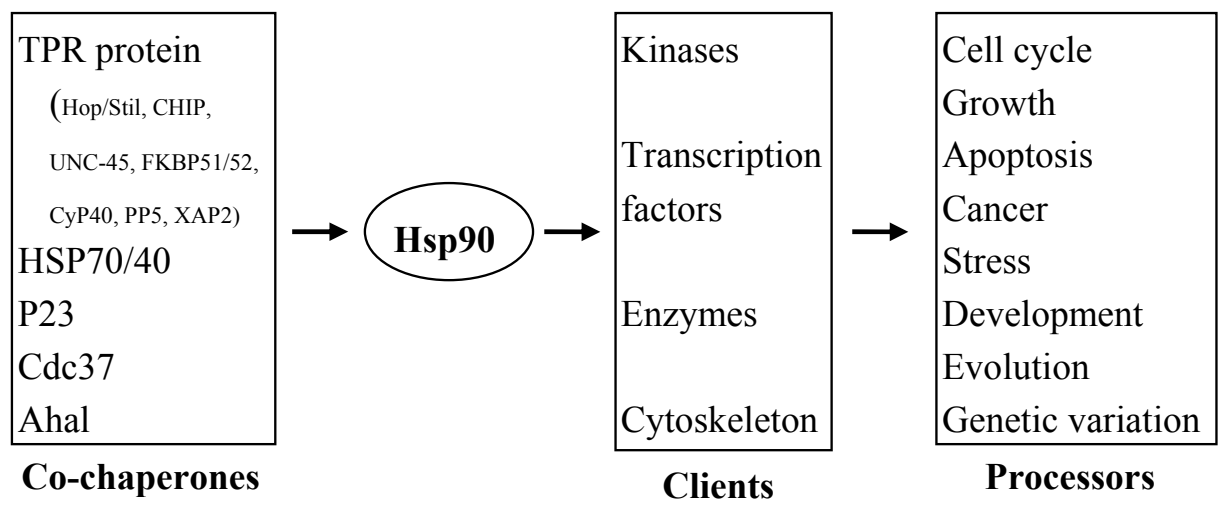

Table 1. Proteins interacting with Hsp90s.

\begin{tabular}{ll}
\hline Chaperones and relatives: & Transcription factors: \\
\hline - Cdc37 (= p50) & -12 (S)-HETE receptor \\
- Cdc37 relative Harc & - Achlya steroid(antheridiol) receptor \\
- Hsp70 & - All vertebrate steroid receptors \\
- Human DnaJ homolog Hsj1b & (glucocorticoid, mineralocorticoid, \\
- p23/Sba1 & androgen, progesterone, and estrogen \\
- Proteins with tetratricopeptide motifs: Hop/Sti1, & receptors) \\
FKBP51, FKBP52 (+ high MW plant homologs), & - cytoplasmic v-erbA \\
cyclophilin-40/Cpr6 and Cpr7, protein phosphatase & - Hap1 \\
5, Tom70, Ah receptor interacting protein AIP, Cns1 & - Heat-shock transcription factor HSF-1 \\
(and its Drosophila relative Dpit47), CHIP, & - p53 \\
UNC-45/She4 & - PAS family members: Dioxin receptor \\
- Sse1 & (= AhR), Sim and HIF-1a \\
\hline
\end{tabular}


Table 1. Cont.

\begin{tabular}{ll}
\hline Kinases: & Others: \\
\hline - Akt (= protein kinase B) & - Actin, tubulin, myosin \\
- Bcr-Abl & - Apaf-1 \\
- Casein kinase II $a$ catalytic subunit & - apoB \\
- Cdk4, Cdk6, Cdk9 & - Atrial natriuretic peptide receptor \\
- c-Mos & - Calcineurin/Cna2 (catalytic subunit) \\
- Death domain kinase RIP & - Calmodulin \\
- eEF-2 kinase & - Calponin \\
- eIF2- $a$ kinases HRI, PKR, Gcn2 & - Ctf13/Skp1 component of CBF3 \\
- ErbB2 & - DNA polymerase $a$ \\
- IkB kinases $a$ and $b$ & - eNOS, nNOS \\
- Insulin receptor & - Free $b g$ subunit of G protein \\
- KSR & - Ga0, Ga 12 \\
- MEK & - GERp95 \\
- Mik1 & - Macromolecular aminoacyl-tRNA synthetase \\
- MOK, MAK, MRK & complex \\
- Nucleophosmin-anaplastic lymphoma kinase & - Macrophage scavenger receptor \\
- PDK-1 & - Mdm2 \\
- Pim-1 & - Nascent CFTR \\
- Plk1 & - Proteasome \\
- pp60v-src, c-src & - Rreovirus protein $s 1$ \\
- src related tyrosine kinases: yes, fps, fes, fgr, & - Reverse transcriptase of hepatitis B virus \\
hck, and lck & - SV40 large T-antigen \\
- Raf-1, B-Raf, Ste11 & - Telomerase \\
- Wee1, Swe1 & - Thrombin receptor (PAR-1) \\
\hline & - Vaccina core protein 4a \\
\hline &
\end{tabular}

\subsection{Regulators}

Hsp90s serve as chaperones depending on phosphorylation and stimulate heme to regulate HRI under phosphorylation by casein kinase II or secondary phosphorylation [47]. Phosphorylation of Hsp90s induces the release of substrate proteins, such as pp60v-src and reovirus protein $\sigma 1$ [48]. Sch9 kinase, which inhibits the function of Hsp90 in yeast [49], cooperated to regulate PKA activity [50]. Treatment with the Hsp90 inhibitor radicicol upregulated 114 genes in yeast, and among them 26 contained Msn2/Msn4 binding elements [51]. More recently, a report demonstrated that an Hsp90 mutation selectively disrupted cAMP/PKA signaling in Saccharomyces cerevisiae, indicating that a specific interaction of Hsp90 and Sgt1 with Cyr1 plays a key role in regulating gene expression [52]. However, the phosphorylation sites of Hsp90s and the molecular mechanism of most kinases have yet to be elucidated.

\subsection{Substrate Proteins}

Hsp90s affect the folding and activation of a wide variety of substrate proteins, most of which are kinases and transcription factors involved in signal transduction and regulatory processes $[5,53,54]$. 
Correspondingly, the stabilized conformation of these substrates require HSP90 [55]. It was reported that PKB/AKTs combine with the middle domain of Hsp90s to regulate their kinase activity by proteasome-mediated degradation [56]. Src family members require the participation of Hsp90 to refold them during transportation through membranes. Hsp90 inhibited PKR kinase activated by dsRNA to maintain a mature form of PKR [57]. Recent, research showed that Hsp90-p23 interacted with hTERT (telomerase reverse transcriptase) to regulate the activity of telomerase in maintaining its normal behavior [58]. Additionally, the conformational plasticity of Hsp90s may allow them to adapt to structurally diverse substrates, thereby catalyzing further structural changes that lead to ATP hydrolysis [59]. Folding of most proteins in yeast does not require involvement of Hsp90, but Hsp90 can promote the damage repair of proteins under stress. However, fundamental distinction between proteins dependent and independent of Hsp90 is in need of further research.

Hsp90s in animals are extensively involved in signal transduction, protein synthesis and folding, and DNA repair, functions that highlight their growing significance $[4,5,8,43]$. Now that Hsp90 is a target of the antitumor drug geldanamycin [35], increasing attention is focusing on the group. However, the biological functions of Hsp90s in plants are less well identified. At present, these are supposed to mainly involve assisted protein folding, and protein complex assembly and degradation, as well as activation of substrates or enhancing the biological activity of substrate proteins $[60,61]$.

\section{The Hsp90 Family in Model Plants}

Although Hsp90s have been identified in various plant species, the evolutionary relationships of Hsp90 among them are uncertain. Three representative dicotyledons (Arabidopsis, soybean and grapevine) and two monocotyledons (rice and maize) were selected for study. Four databases were searched to identify Hsp90 genes: viz., TAIR (the Arabidopsis Information Resource) [62], Rice Genome Annotation Project Database [63], Phytozome [64], and Maize Genome Annotation Project Database [65]. When there was more than one allele, the longest was chosen as representative. Seven Hsp90 genes were identified in Arabidopsis (Table 2) [66], and 15, nine, and 12 putative Hsp90 genes were identified in soybean (Table 3), rice (Table 4), and maize (Table 5), respectively. Hsp90 genes in grapevine were analyzed by Banilas et al. [18]. Predictions of the subcellular locations of plant Hsp90 proteins were made using PSORT [67].

Table 2. Arabidopsis Hsp90 proteins.

\begin{tabular}{cccc}
\hline Nomenclature & Amino acids & Chromosome & Intracellular localization \\
\hline AtHSP90.5 & 780 & II & Chloroplast stroma \\
AtHSP90.6 & 799 & III & Mitochondrial matrix space \\
AtHSP90.7 & 823 & IV & Endoplasmic reticulum \\
AtHSP90.1 & 705 & V & Cytoplasm \\
AtHSP90.4 & 699 & V & Nucleus \\
AtHSP90.3 & 668 & V & Nucleus \\
AtHSP90.2 & 728 & V & Nucleus \\
\hline
\end{tabular}


Table 3. Glycine max Hsp90 proteins.

\begin{tabular}{cccc}
\hline Nomenclature & $\begin{array}{c}\text { Amino } \\
\text { acids }\end{array}$ & Chromosome & Intracellular localization \\
\hline Glyma08g44590.1 & 699 & VIII & Nucleus and plasma membrane \\
Glyma18g08220.1 & 702 & XVIII & Nucleus and plasma membrane \\
Glyma14g01530.1 & 700 & XIV & Nucleus and plasma membrane \\
Glyma02g47210.1 & 702 & II & Nucleus and plasma membrane \\
Glyma09g24410.1 & 699 & IX & Nucleus and plasma membrane \\
Glyma16g29750.1 & 699 & XVI & Nucleus and plasma membrane \\
Glyma08g03690.1 & 712 & VIII & Nucleus and plasma membrane \\
Glyma14g40320.1 & 847 & XIV & Endoplasmic reticulum \\
Glyma14g40320.2 & 816 & XIV & Endoplasmic reticulum \\
Glyma14g40320.4 & 727 & XIV & Nucleus and endoplasmic reticulum (lumen) \\
Glyma17g37820.1 & 814 & XVII & Endoplasmic reticulum \\
Glyma02g13790.1 & 794 & II & Chloroplast stroma and mitochondrial matrix space \\
Glyma02g47580.1 & 791 & II & Mitochondrial matrix space and nucleus \\
Glyma01g09310.1 & 793 & I & chloroplast stroma and mitochondrial matrix space \\
Glyma14g01100.1 & 797 & XIV & Mitochondrial matrix space and nucleus \\
\hline
\end{tabular}

Table 4. Oryza sativa Hsp90 proteins.

\begin{tabular}{cccc}
\hline Nomenclature & Amino acids & Chromosome & Intracellular localization \\
\hline Os04g01740.1 & 703 & IV & Nucleus \\
Os06g50300.1 & 812 & VI & Endoplasmic reticulum \\
Os08g38086.3 & 761 & VIII & Mitochondrial matrix space \\
Os08g39140.1 & 699 & VIII & Nucleus \\
Os09g29840.1 & 791 & IX & Mitochondrial matrix space \\
Os09g30412.1 & 699 & IX & Nucleus \\
Os09g30418.1 & 830 & IX & Nucleus \\
Os09g36420.1 & 1046 & IX & Nucleus \\
Os12g32986.1 & 811 & XII & Vacuole \\
\hline
\end{tabular}

Table 5. Zea mays Hsp90 proteins.

\begin{tabular}{cccl}
\hline Nomenclature & Amino acids & Chromosome & \multicolumn{1}{c}{ Intracellular localization } \\
\hline Zm2G012631_T01 & 699 & II & Nucleus and mitochondrial matrix space \\
Zm2G112165_T01 & 698 & II & Nucleus and mitochondrial matrix space \\
Zm2G069651_T01 & 699 & II & Nucleus and mitochondrial matrix space \\
Zm5G833699_T01 & 714 & V & Nucleus and mitochondrial matrix space \\
Zm2G141931_T01 & 804 & II & Endoplasmic reticulum and vacuole \\
Zm2G141931_T03 & 710 & II & Endoplasmic reticulum and vacuole \\
Zm2G399073_T02 & 1001 & II & Endoplasmic reticulum \\
Zm2G399073_T03 & 808 & II & Endoplasmic reticulum \\
Zm2G141931_T04 & 667 & II & Endoplasmic reticulum \\
& & & Chloroplast stroma and chloroplast \\
Zm2G002220_T01 & 793 & II & thylakoid membrane \\
Zm5G813217_T01 & 758 & V & Nucleus and chloroplast stroma \\
Zm5G813217_T03 & 708 & V & Nucleus and mitochondrial matrix space \\
\hline
\end{tabular}


Figure 4. Phylogenetic tree of Hsp90 genes from model plants. Alignments of protein sequences were performed using the CLUSTALW program [68] with default parameters. The rectangular phylogram of the Hsp90 protein sequences was generated using MEGA 4.1 [69]. Abbreviations: AT, Arabidopsis thaliana; Os, Oryza sativa; Glyma, Glycine max; Zm, Zea mays.

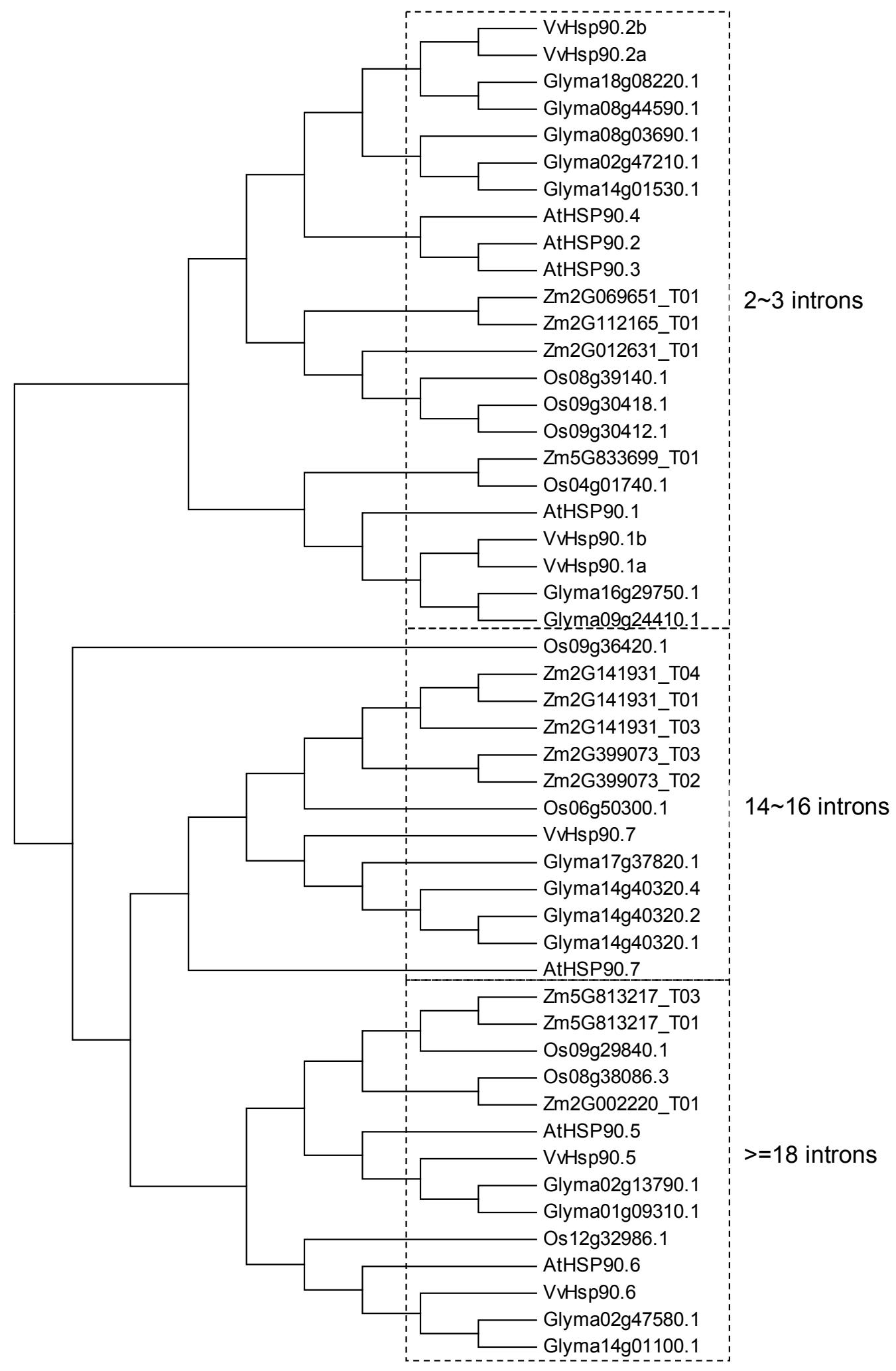


We performed multiple alignment analyses using full-length amino acid sequences. The alignments indicated that Hsp90s could be placed in 3 clusters (I to III) and 10 sub-clusters according to their phylogenetic relationships, gene structures, conserved motifs, and biological functions (Figure 4). Gene structures of clusters I, II, and III were characterized by having more than 18, 14 to 16, and 2 to 3 introns, respectively, suggesting that intron numbers were established in each cluster before divergence of dicotyledons and monocotyledons.

Homologous genes from the dicotyledons shared high homology, and likewise the monocotyledons, indicating that the Hsp90 genes evolved further in species-specific ways, although gene structure organization and expression characteristics of Hsp90s are similar in different plants. However, the relationships of homologs are less clear. For example, rice Os12g32986.1 was homologous to Arabidopsis At3G07770, soybean Glyma02g47580.1 and Glyma14g01100.1, but Os12g32986.1 protein was localized in the vacuole whereas its counterparts from Arabidopsis and soybean (At3G07770, Glyma02g47580.1, and Glyma14g01100.1) accumulated in the mitochondrial matrix space and nucleus, respectively.

Over the past decade, investigation of Arabidopsis Hsp90s has attracted the most attention. AtHSP90-2, -3, and -4 have high similarity with homology of about 96\%, implying functional redundancy. AtHSP90-1, -2, -3, and -4 contain the specific target signal MEEVD, essential for sub-cellular location in the cytoplasm, at the $C$-terminal. AtHsp90-5, -6, and -7 were predicted to be located within the plastidial, mitochondrial, and endoplasmic reticulum compartments, respectively, based on their $N$-terminal signal peptides [20,55].

Loss of Hsp90 function in plants leads to deformities and affects physiological characteristics [4,5]. Inhibition in Arabidopsis resulted in an abnormal plant phenotype, including an epinastic cotyledon, disc or radial symmetry of cotyledons, and abnormal growth of root hairs [70]. Hsp90-RNAi lines had increased accumulation of purple pigment in the cotyledons with later development of true, but narrower leaves. The developing plants lacked apical dominance, and produced multiple primary inflorescences with abnormal stems and increased numbers of rosette leaves. They were also later flowering [71].

Hsp90 is also involved in embryo development and seed germination. A dramatic increase in AtHsp90-1 transcript was observed in embryos during their development, whereas AtHsp90-3 increased slowly during pod elongation and decreased slightly in nearly mature embryos. It was concluded that Hsp90 participated in the process of seed embryo formation and seed germination [72]. Hsp90 also affected elongation of the hypocotyl [73]. Also, it was recently shown that constitutive overexpression of AtHsp90-3 resulted in lower germination rate and shorter root length in Arabidopsis [74]. Thus, Hsp90 appears to be extensively involved in plant growth and development.

\section{Hsp90 Is Involved in Plant Stress Resistance}

Hsp90 are usually maintained in the dipolymer form and are sharply induced under extreme conditions. Many Hsps, including Hsp90, are induced by heat shock stress and are involved in regulation of heat shock response. There are several heat shock elements (HSEs) bound with heat shock factors (HSFs) in the promoters of Hsps. In normal conditions, the HSF monomer is combined with Hsp90 dimers; but under stress conditions, the HSF monomer converts to a trimer and closely 
integrates with HSE to initiate transcription of Hsp genes [21,75]. HSP is induced not only by heat shock, but also by many other stresses.

It seems that most of the substrates of Hsp90s are kinases and transcription factors that function pivotally in signal transduction to activate or suppress defense gene expression, as well as in the regulation of interaction between different signaling pathways [76]. Clearly, Hsp90s mediate both abiotic and biotic stress resistances in plants.

\subsection{Hsp90s Are Involved in Disease and Pest Resistances}

In recent years, it has become apparent that Hsp90s mediate signal transduction pathways of disease resistance in plants. Plant immunity is initiated by resistance $(\mathrm{R})$ proteins that recognize pathogen effector proteins. The interaction of Hsp90 and SGT1 (suppressor of G2 allele kinetochore protein) and RAR1 (required for Mla12 resistance) confers stability to R protein contributing to the recognition of pathogen eggectors. SGT1 interacts with Hsp90 through its CS domain and TPR domain binding with the ATPase site and $C$-terminal of Hsp90, respectively, and SGT1 binds with the R protein at the LRR (leucine rich repeat) domain, while RAR1 recognizes the ATP binding region of Hsp90 [77]. Hsp90 complexes in Arabidopsis directly regulate the resistance protein activity and play a key role in assisting in R-mediated disease resistance [78]. Disease resistance mediated by RPM1 was weakened in AtHsp90-2 mutant plants [79].

Hsp90 is required for regulation of resistance to Pseudomonas cloves conferred by the tomato Pto gene [80]. The interaction of Hsp90 with RAR1 and TIR-NB-LRR in tobacco improved mosaic virus (TMV) resistance in tobacco [81]. It was proved through BSMV-VIGS technology that the SGTl, RAR1, and Hsp90 genes are necessary for Mla13-mediated powdery mildew resistance in barley and Lr21-mediated leaf rust resistance in wheat [82-84].

Recent studies revealed that Hsp90 are also involved in signal transduction pathways of plant resistance to insects. Tomato Mi-1 protein containing a nucleotide binding site (nucleotide-binding site, NBS) and LRR motifs mediate resistance to root-knot nematodes, potato aphid and whitefly. Knockouts of SGT1 and Hsp90 weakened the Mi-1-mediated resistance to root-knot nematode and potato aphid, whereas RARl was not necessary [85]. The mechanisms involved in these responses require further detailed study.

\subsection{Hsp90s Mediate Abiotic Stress Resistance}

Hsp90s mediate plant abiotic stress signal pathways [86], but again the mechanisms are unclear. A Hsp90-specific inhibitor in the cytoplasm induced the accumulation of heat-induced gene products and improved heat tolerance in Arabidopsis [87,88]. Under normal conditions, Hsp90.2 in the cytoplasm negatively regulates transcription of heat-induced genes by suppression of HSF. Hsp90.2 is instantaneously inactivated under heat shock, and HSF is activated to induce expression of downstream genes containing HSF elements [87]. It was subsequently confirmed that overexpression of Hsp90.2 suppresses HsfA2 transcription, and that HsfA2 is induced under inhibition of Hsp90.2 in order to adapt to oxidative stress [88]. Similarly, an inhibitor of Hsp90 produced by a rhizosphere fungus repressed the growth and development of plants, but enhanced the heat resistance of 
Arabidopsis [89]. These studies show that Hsp90s play important roles in adaptation of plants to stress environments.

Although Hsp90s are involved in abiotic stress responses in plants, little research on improving abiotic stress resistance has been reported. The expression of UpHSP90 was strongly and positively regulated by diurnal and temperature changes, and slightly influenced by long-term treatment with heavy metal stress in sterile Ulva pertusa [90]. Overexpression of $r H s p 90$ from rice significantly increased salt stress in tobacco [91]. Yeast Hsp90 was involved in yeast cell wall permeability and stress response through the HOG and MAPK pathways [92]. The MAPK signal pathway occurs in plants. Recent study showed that the MAPK cascade functions down-stream of Hsp90 and transduces the cell death signal to mitochondria for $N$ gene-dependent cell death [93].

The way by which auxiliary proteins regulate the ATPase activity of Hsp90 in cytoplasm to assist Hsp90 to interact with the substrates is similar in animals and plants. Arabidopsis FKBP (FK506 binding protein) family members ROF1 (FKBP62) interact with Hsp90.1 through the TPR domain [41]. Under normal conditions the ROF1-Hsp90.1 complex targets the cytoplasm. When stimulated by heat, HsfA2 combines with the complex to form a ROF1-Hsp90.1-HsfA2 complex that migrates to the nucleus and regulates sHsp transcription to enhance heat tolerance [94]. Silencing of ROF1 or ROF2 (FKBP65, ROF1 homologous protein) increases the heat tolerance of plants, but ROF2 nuclear localization is independent of Hsp90.1 or HsfA2 which negatively regulate activation of HsfA2 and inhibit transcription of sHsp expression [95].

\section{Prospects}

In animals and fungi, Hsp90s generally mediate stress signal transduction, folding of transcription factors and protein kinases. High expression of human Hsp90 is closely related to tumor development and may enhance the anti-apoptosis capacity of tumor cells, possibly offering a new therapy for treatment of cancer. The interactions of numerous proteins with Hsp90s were discovered only recently. These interactions provide opportunities for in-depth study of the functions and mechanisms of Hsp90s. Similarly, plant Hsp90s are involved in a variety of biological processes related to growth and development, as well as various responses to environmental stress. In Arabidopsis, overexpression of AtHsp 90.2, AtHsp90.5 or AtHsp90.7 reduced plant tolerance to drought stress [96]. When grapevine was suffered from drought combined with heat stress, the up-regulation of $V v H s p 901 a$ was delayed [18]. These results provide insights into the roles of Hsp90 machinery in plant development and response to adversity stress, and promote further research to better understand the molecular mechanisms of plant response to environmental stress.

Knowledge of Hsp90 in plants is much less advanced and there are fewer reports about the physiological functions of Hsp90 chaperone complexes. Although the results of recent studies revealing that Hsp90s could improve plant disease resistance and buffer genetic variation have gained attracted the interest of plant biologists, the potential roles of plant Hsp90s are still not clear; especially the characteristics of all plant Hsp90s and the Hsp90-mediated signal pathways. It is of theoretical and practical significance that in-depth investigation of the molecular relationship among Hsp90s, environmental stresses, and resistance in plants may contribute to molecular breeding for stress resistance. 


\section{Acknowledgments}

This research was financially supported by the National Natural Science Foundation of China (31171546). We are grateful to RA McIntosh (Plant Breeding Institute, University of Sydney) for critically reading the manuscript.

\section{References}

1. Lindquist, S. The heat-shock response. Annu. Rev. Biochem. 1986, 55, 1151-1191.

2. Lindquist, S.; Craig, E.A. The heat-shock proteins. Annu. Rev. Genetics 1988, 22, 631-677.

3. Horwitz, J. Alpha-crystallin can function as a molecular chaperone. Proc. Natl. Acad. Sci. USA 1992, 89, 10449-10453.

4. Picard, D. Heat-shock protein 90, a chaperone for folding and regulation. Cell. Mol. Life Sci. 2002, 59, 1640-1648.

5. Jackson, S.E.; Queistch, C.; Toft, D. Hsp90: From structure to phenotype. Nat. Struct. Mol. Biol. 2004, 11, 1152-1155.

6. Wegele, H.; Muller, L.; Buchner, J. Hsp70 and Hsp90-a relay team for protein folding. Rev. Physiol. Biochem. Pharmacol. 2004, 151, 1-44.

7. Shinozaki, F.; Minami, M.; Chiba, T.; Suzuki, M.; Yoshimatsu, K.; Ichikawa, Y.; Terasawa, K.; Emori, Y.; Matsumoto, K.; Kurosaki, T.; et al. Depletion of Hsp90ß induces multiple defects in B cell receptor signaling. J. Biol. Chem. 2006, 281, 16361-16369.

8. Zuehlke, A.; Johnson, J.L. Hsp90 and co-chaperones twist the functions of diverse client proteins. Biopolymers 2010, 93, 211-217.

9. Scroggins, B.T.; Robzyk, K.; Wang, D.; Marcu, M.G.; Tsutsumi, S.; Beebe, K.; Cotter, R.J.; Felts, S.; Toft, D.; Karnitz, L.; et al. An acetylation site in the middle domain of Hsp90 regulates chaperone function. Mol. Cell 2007, 25, 151-159.

10. Kamal, A.; Thao, L.; Sensintaffar, J.; Zhang, L.; Boehm, M.F.; Fritz, L.C.; Burrows, F.J. A high-affinity conformation of Hsp90 confers tumour selectivity on Hsp90 inhibitors. Nature 2003, $425,407-410$.

11. Xu, W.; Neckers, L. Targeting the molecular chaperone Heat Shock Protein 90 provides a multifaceted effect on diverse cell signaling pathways of cancer cells. Clin. Cancer Res. 2007, 13, 1625-1629.

12. Ali, M.M.; Roe, S.M.; Vaughan, C.K.; Meyer, P.; Panaretou, B.; Piper, P.W.; Prodromou, C.; Pearl, L.H. Crystal structure of an Hsp90-nucleotide-p23/Sba1 closed chaperone complex. Nature 2006, 440, 1013-1017.

13. Chen, Z.; Sasaki, T.; Tan, X.; Carretero, J.; Shimamura, T.; Li, D.; Xu, C.; Wang, Y.; Aldelmant, G.O.; Capelletti, M.; et al. Inhibition of ALK, PI3K/MEK and HSP90 in murine lung adenocarcinoma induced by EML4-ALK fusion oncogene. Cancer Res. 2010, 70, 9827-9836.

14. Grigorova, B.; Vaseva, I.; Demirevska, K.; Feller, U. Combined drought and heat stress in wheat: Changes in some heat shock proteins. Biol. Plant 2011, 55, 105-111.

15. Maksymied, W. Effects of jasmonates and some other signaling factors on bean and onion growth during the initial phase of cadmium action. Biol. Plant 2011, 55, 112-118. 
16. Wójcik, M.; Tukiendorf, A. Glutathione in adaptation of Arabidopsis thaliana to cadmium stress. Biol. Plant 2011, 55, 125-132.

17. Zhang, L.; Fan, Y.; Shi, F.; Qin, S.; Liu B. Moleculat cloning, characterization, and expression analysis of a cytosolic HSP90 gene from Haematococcus pluvialis. J. Appl. Phycol. 2012, doi:10.1007/s10811-012-9821-5.

18. Banilas, G.; Korkas, E.; Englezos, V.; Nisiotou, A.A.; Hatzopoulos, P. Genome-wide analysis of the heat shock protein 90 gene family in grapevine (Vitis vinifera L.). Aust. J. Grape Wine Res. 2012, 18, 29-38.

19. Rizhsky, L.; Liang, H.; Mittler, R. The combined effect of drought stress and heat shock on gene expression in tobacco. Plant Physiol. 2002, 130, 1143-1151.

20. Sangster, T.A.; Queitsch, C. The HSP90 chaperone complex, an emerging force in plant development and phenotypic plasticity. Curr. Opin. Plant Biol. 2005, 8, 86-92.

21. Lindquist, S.; Jarosz, D.F. Hsp90 and environmental stress transform the adaptive value of natural genetic variation. Science 2010, 330, 1820-1824.

22. Sangster, T.A.; Salathia, N.; Lee, H.N.; Watanabe, E.; Schellenberg, K.; Morneau, K.; Wang, H.; Undurraga, S.; Queitsch, C.; Lindquist, S. HSP90-buffered genetic variation is common in Arabidopsis thaliana. Proc. Natl. Acad. Sci. USA 2008, 105, 2969-2974.

23. Young, J.C.; Moarefi, I.; Hartl, F.U. Hsp90: A specialized but essential protein-folding tool. J. Cell Biol. 2001, 154, 267-273.

24. Gupta, R.S. Phylogenetic analysis of the $90 \mathrm{kD}$ heat shock family of protein sequences and an examination of the relationship among animals, plant, and fungi species. Mol. Biol. Evol. 1995, 12, 1063-1073.

25. Hao, H.; Naomoto, Y.; Bao, X.; Watanabe, N.; Sakurama, K.; Noma, K.; Motoki, T.; Tomono, Y.; Fukazawa, T.; Shirakawa, Y.; et al. HSP90 and its inhibitors. Oncol. Rep. 2010, 23, 1483-1492.

26. Ogiso, H.; Kagi, N.; Matsumoto, E.; Nishimoto, M.; Arai, R.; Shirouzu, M.; Mimura, J.; Fujii-Kuriyama, Y.; Yokoyama, S. Phosphorylation analysis of $90 \mathrm{kDa}$ heat shock protein within the cytosolic arylhydrocarbon receptor complex. Biochemistry 2004, 43, 15510-15519.

27. Parsell, D.A.; Lindquist, S. The function of heat-shock proteins in stress tolerance: Degradation and reactivation of damaged proteins. Annu. Rev. Genet. 1993. 27, 437-496.

28. Csermely, P.; Schnaider, T.; Soti, C.; Prohaszka, Z.; Nardai, G. The 90-kDa molecular chaperone family: Structure, function, and clinical applications. A comprehensive review. Pharmacol. Ther. 1998, 79, 129-168.

29. Terasawa, K.; Minami, M.; Minami, Y. Constantly updated knowledge of Hsp90. J. Biochem. 2005, 137, 443-447.

30. Cowen, L.E. The evolution of fungal drug resistance: Modulating the trajectory from genotype to phenotype. Nat. Rev. Microbiol. 2008, 6, 187-198.

31. Wayne, N.; Mishra, P.; Bolon, D.N. Hsp90 and client protein maturation. Methods Mol. Biol. 2011, 787, 33-44.

32. Hessling, M.; Richter, K.; Buchener, J. Dissection of the ATP-induced conformational cycle of the molecular chaperone Hsp90. Nat. Struct. Mol. Biol. 2009, 16, 287-293.

33. Mickler, M.; Hessling, M.; Ratzke, C.; Buchner, J.; Hugel, T. The large conformational changes of Hsp90 are only weakly coupled to ATP hydrolysis. Nat. Struct. Mol. Boil. 2009, 16, 281-286. 
34. Wegele, H.; Wandinger, S.K.; Schmid, A.B.; Reinstein, J.; Buchner, J. Substrate transfer from the chaperone Hsp70 to Hsp90. J. Mol. Biol. 2006, 356, 802-811.

35. Whitesell, L.; Shifrin, S.D.; Schwab, G.; Neckers, L.M. Benzoquinonoid ansamycins possess selective tumoricidal activity unrelated to src kinase inhibition. Cancer Res. 1992, 52, 1721-1728.

36. Weikl, T.; Muschler, P.; Richter, K.; Veit, T.; Reinstein, J.; Buchner, J. C-terminal regions of Hsp90 are important for trapping the nucleotide during the ATPase cycle. J. Mol. Biol. 2000, 303, 583-592.

37. Meyer, P.; Prodromou, C.; Hu, B.; Vaughan, C.; Roe, S.M.; Panaretou B.; Piper, P.W.; Pearl, L.H. Structural and functional analysis of the middle segment of Hsp90: Implications for ATP hydrolysis and client protein and cochaperone interactions. Mol. Cell 2003, 11, 647-658.

38. Pearl, L.H.; Prodromou, C. Structure and in vivo function of Hsp90. Curr. Opin. Struct. Biol. 2000, 10, 46-51.

39. Zhao, R.; Davey, M.; Hsu, Y.C.; Kaplanek, P.; Tong, A.; Parsons, A.B.; Krogan, N.; Cagney, G.; Mai, D.; Greenblatt, J.; et al. Navigating the chaperone network: An integrative map of physical and genetic interactions mediated by the hsp90 chaperone. Cell 2005, 120, 715-727.

40. Johnson, J.L.; Brown, C. Plasticity of the Hsp90 chaperone machine in divergent eukaryotic organisms. Cell Stress Chaperon 2009, 14, 83-94.

41. Aviezer-Hagai, K.; Skovorodnikova, J.; Galigniana, M.; Farchi-Pisanty, O.; Maayan, E.; Bocovza, S.; Efrat. Y.; von Koskull-Döring, P.; Ohad, N.; Breiman, A. Arabidopsis immunophilins ROF1 (AtFKBP62) and ROF2 (AtFKBP65) exhibit tissue specificity, are heat-stress induced, and bind HSP90. Plant Mol. Biol. 2007, 63, 237-255.

42. Weaver, A.J.; Sullivan, W.P.; Felts, S.J.; Owen, B.A.; Toft, D.O. Crystal structure and activity of human p23, a heat shock protein 90 co-chaperone. J. Mol. Biol. 2000, 275, 23045-23052.

43. Tinel, A.; Eckert, M.J.; Logette, E.; Lippens, S.; Janssens, S.; Jaccard, B.; Quadroni, M.; Tschopp, J. Regulation of PIDD auto-proteolysis and activity by the molecular chaperone Hsp90. Cell Death Differ. 2010, 18, 506-515.

44. Janssens, S.; Tinel, A.; Lippens, S.; Tschopp, J. PIDD mediates NF- $\kappa$ B activation in response to DNA damage. Cell 2005, 123, 1079-1092.

45. Hunter, T.; Poon, R.Y.C. Cdc37: A protein kinase chaperone. Trends Cell. Biol. 1997, 7, 157-161.

46. Dmochewitz, L.; Lillich, M.; Kaiser, E.; Jennings, L.D.; Lang, A.E.; Buchner, J.; Fischer, G.; Aktories, K.; Collier, R.J.; Barth, H. Role of CypA and Hsp90 in membrane translocation mediated by anthrax protective antigen. Cell. Microbiol. 2010, 13, 359-373.

47. Szyszka, R.; Kramer, G.; Hardesty, B. The phosphorylation state of the reticulocyte 90-kDa heat shock protein affects its ability to increase phosphorylation of peptide initiation factor 2a subunit by the heme-sensitive kinase. Biochemistry 1989, 28, 1435-1438.

48. Mimnaugh, E.G.; Worland, P.J.; Whitesell, L.; Neckers, L.M. Possible role for serine/threonine phosphorylation in the regulation of the heteroprotein complex between the hsp90 stress protein and the pp60v-src tyrosine kinase. J. Biol. Chem. 1995, 270, 28654-28659.

49. Morano, K.A.; Thiele, D.J. The Sch9 protein kinase regulates Hsp90 chaperone complex signal transduction activity in vivo. EMBO J. 1999, 18, 5953-5962. 
50. Trott, A.; Shaner, L.; Morano, K.A. The molecular chaperone Sse1 and the growth control protein kinase Sch9 collaborate to regulate protein kinase A activity in Saccharomyces cerevisiae. Genetics 2005, 107, 1009-1021.

51. Echeverria, P.C.; Flrafonov, F.; Pandey, D.P.; Muhlebach, G.; Picard, D. Detection of changes in gene regulatory patterns, elicited by perturbations of the Hsp90 molecular chaperone complex, by visualizing multiple experiments with an animation. BioData Min. 2011, 4, 15.

52. Flom, G.A.; Langner, E.; Johnson, J.L. Identification of an Hsp90 mutation that selectively disrupts cAMP/PKA signaling in Saccharomyces cerevisiae. Curr. Genet. 2012, doi:10.1007/s00294-012-0373-7.

53. Pratt, W.B.; Galigniana, M.D.; Harrell, J.M.; DeFranco, D.B. Role of hsp90 and the hsp90-binding immunophilins in signalling protein movement. Cell Signal. 2004, 16, 857-872.

54. Krukenberg, K.A.; Street, T.O.; Lavery, L.A.; Agard, D.A. Conformational dynamics of the molecular chaperone Hsp90. Q. Rev. Biophys. 2011, 44, 229-255.

55. Milioni, D.; Hatzopoulos, P. Genonic organization of hsp90 gene family in Arabidopsis. Plant Mol. Biol. 1997, 35. 955-961.

56. Sato, S.; Fujita, N.; Tsuruo, T. Modulation of Akt kinase activity by binding to Hsp90. Proc. Natl. Acad. Sci. 2000, 97, 10832-10837.

57. Bijlmakers, M.J.; Marsh, M. Hsp90 is essential for the synthesis and subsequent membrane association, but not the maintenance, of the Src-kinase p561ck. Mol. Biol. Cell 2000, 11, 1585-1595.

58. Lee, J.H.; Chung, I.K. Curcumin inhibits nuclear localization of telomerase by dissociating the Hsp90 co-chaperone p23 from hTERT. Cancer Lett. 2010, 290, 76-86.

59. Street, T.O.; Lavery, L.A.; Agard, D.A. Substrate binding drives large-scale conformational changes in the Hsp90 molecular chaperone. Mol. Cell 2011, 42, 96-105.

60. Samakovli, D.; Thanou, A.; Hztzopoulos, P. Hsp90 canalizes developmental perturbation. J. Exp. Bot. 2007, 58, 3513-3524.

61. Specchia, V.; Piacentini, L.; Tritto, P.; Fanti, L.; D’Alessandro, R.; Palumbo, G.; Pimpinelli, S.; Bozzetti, M.P. Hsp90 prevents phenotypic variation by suppressing the mutagenic acivity of transposons. Nature 2010, 463, 662-665.

62. TAIR. Available online: http://www.arabidopsis.org (accessed on 13 November 2012).

63. Plant Biology. Available online: http://www.rice.plantbiology.msu.edu (accessed on 13 November 2012).

64. Phytozome. Available online: http://www.phytozome.net/soybean (accessed on 13 November 2012).

65. MaizeGDB. Available online: http://www.maizegdb.org (accessed on 13 November 2012).

66. Krishna, P.; Gloor, G. The Hsp90 family of proteins in Arabidopsis thaliana. Cell Stress Chaperon 2001, 6, 238-246.

67. PSORT. Available online: http://psort.nibb.ac.jp/form.html (accessed on 13 November 2012).

68. Thompson, J.D.; Gibson, T.J.; Higgins, D.G. Multiple sequence alignment using ClustalW and ClustalX. Curr. Protoc. Bioinforma. 2002, 2.3.1-2.3.22.

69. Tamura, K.; Dudley, J.; Nei, M.; Kumar, S. MEGA4: Molecular Evolutionary Genetics Analysis (MEGA) software version 4.0. Mol. Biol. Evol. 2007, 24, 1596-1599. 
70. Queitsch, C.; Sangster, T.A.; Lindquist, S. Hsp90 as a capacitor of phenotypic variation. Nature 2002, 417, 598-599.

71. Sangster, T.A.; Bahrami, A.; Wilczek, A.; Watanabe, E.; Schellenberg, K.; McLellan, C.; Kelley, A.; Kong, S.W.; Queitsch, C.; Lindquist, S. Phenotypic diversity and altered environmental plasticity in Arabidopsis thaliana with reduced Hsp90 levels. PLoS One 2007, 2, e648.

72. Prasinos, C.; Krampis, K.; Samakovli, D.; Hatzopoulos, P. Tight regulation of expression of two Arabidopsis cytosolic HSP90 genes during embryo development. J. Exp. Bot. 2005, 56, 633-644.

73. Sangster, T.A.; Salathia, N.; Undurraga, S.; Milo, R.; Schellenberg, K.; Lindquist, S.; Queitsch, C. HSP90 affects the expression of genetic variation and developmental stability in quantitative traits. Proc. Natl. Acad. Sci. USA 2008, 105, 2963-2968.

74. Song, H.M.; Wang, H.Z.; Xu, X.B. Overexpression of AtHsp90.3 in Arabidopsis thaliana impairs plant tolerance to heavy metal stress. Biol. Plant 2012, 56, 197-199.

75. Lohmann, C.; Eggers-Schumacher, G.; Wunderlich, M.; Schöffl, F. Two different heat shock transcription factors regulate immediate early expression of stress genes in Arabidopsis. Mol. Genet. Genomics 2004, 271, 11-21.

76. Xu, Z.S.; Chen, M.; Li, L.C.; Ma, Y.Z. Functions and application of the AP2/ERF transcription factor family in crop improvement. J. Integr. Plant Biol. 2011, 53, 570-585.

77. Shirasu, K.; Schulze-Lefert, P. Complex formation, promiscuity and multi-functionality: Protein interactions in disease-resistance pathways. Trends Plant Sci. 2003, 8, 252-258.

78. Takahashi, A.; Casais, C.; Ichimura, K.; Shirasu, K. HSP90 interacts with RAR1 and SGT1, and is essential for RPS2-mediated disease resistance in Arabidopsis. Proc. Natl. Acad. Sci. USA 2003, 100, 11777-11782.

79. Hubert, D.A.; Tornero, P.; Belkhadir, Y.; Krishna, P.; Takahashi, A.; Shirasu, K.; Dangl, J.L. Cytosolic HSP90 associates with and modulates the Arabidopsis RPM1 plant disease resistance protein. EMBO J. 2003, 22, 5679-5689.

80. Lu, R.; Malcuit, I.; Moffett, P.; Ruiz, M.T.; Peart, J.; Wu, A.J.; Rathjen, J.P.; Bendahmane, A.; Day, L.; Baulcombe, D.C. High throughput virus-induced gene silencing implicates heat shock protein 90 in plant disease resistance. EMBO J. 2003, 22, 5690-5699.

81. Liu, Y.; Burch-Smith, T.; Schiff, M.; Feng, S.; Dinesh-Kumar, S.P. Molecular chaperone Hsp90 associates with resistance protein $\mathrm{N}$ and its signaling proteins SGT1 and Rar1 to modulate an innate immune response in plants. J. Biol. Chem. 2004, 279, 2101-2108.

82. Hein, I.; Barciszewska-Pacak, M.; Hrubikova, K.; Williamson, S.; Dinesen, M.; Soenderby, I.E.; Sundar, S.; Jarmolowski, A.; Shirasu, K.; Lacomme, C. Virus-induced gene silencing-based functional characterization of genes associated with powdery mildew resistance in barley. Plant Physiol. 2005, 138, 2155-2164.

83. Scofield, S.R.; Huang, L.; Brandt, A.S.; Gill, B.S. Development of a virus-induced gene-silencing system for hexaploid wheat and its use in functional analysis of the Lr21-mediated leaf rust resistance pathway. Plant Physiol. 2005, 138, 2165-2173.

84. Wang, G.F.; Wei, X.; Fan, R; Zhou, H; Wang, X.; Yu, C.; Dong, L.; Dong, Z.; Wang, X.; Kang, Z.; et al. Molecular analysis of common wheat genes encoding three types of cytosolic heat shock protein 90 (Hsp90): Functional involvement of cytosolic Hsp90s in the control of wheat seedling growth and disease resistance. New Phytol. 2011, 191, 418-431. 
85. Bhattarai, K.K.; Li, Q.; Liu, Y.; Dinesh-Kumar, S.P.; Kaloshioan, I. The Mi-1-mediated pest resistance requires Hsp90 and Sgt1. Plant Physiol. 2007, 144, 312-323.

86. Liu, D.L.; Zhang, X.X.; Cheng, Y.X.; Takano, T.; Liu, S.K. Cloning and characterization of the rHsp90 gene in rice (Oryza sativa. L) under environmental stress. Mol. Plant Breed. 2006, 4 , 317-322.

87. Yamada, K.; Fukao, Y.; Hayashi, M.; Fukazawa, M.; Suzuki, I.; Nishimura, M. Cytosolic HSP90 regulates the heat shock response that is responsible for heat acclimation in Arabidopsis thaliana. J. Biol. Chem. 2007, 282, 37794-37804.

88. Nishizawa-Yokoi, A.; Tainaka, H.; Yoshida, E.; Tamoi, M.; Yabuta, Y.; Shigeoka, S. The 26S proteasome function and Hsp90 activity involved in the regulation of HsfA2 expression in response to oxidative stress. Plant Cell Physiol. 2010, 51, 486-496.

89. McLellan, C.A.; Turbyville, T.J.; Wijeratne, E.M.; Kerschen, A.; Vierling, E.; Queitsch, C.; Whitesell, L.; Gunatilaka, A.A. A rhizosphere fungus enhances Arabidopsis thermotolerance through production of an HSP90 inhibitor. Plant Physiol. 2007, 145, 174-182.

90. Tominaga, H.; Coury, D.A.; Amano, H.; Miki, W.; Kakinuma, M. cDNA cloning and expression analysis of two heat shock protein genes, Hsp90 and Hsp60, from a sterile Ulva pertusa (Ulvales, Chlorophyta). Fish Sci. 2012, 78, 415-429.

91. Liu, D.; Zhang, X.; Cheng, Y.; Takano, T.; Liu, S. rHsp90 gene expression in response to several environmental stresses in rice (Oryza sativa L.). Plant Physiol. Bioch. 2006, 44, 380-386.

92. Hawle, P.; Horst, D.; Bebelman, J.P.; Yang, X.X.; Siderius, M.; van der Vies, S.M. Cdc37p is required for stress-induced high-osmolarity glycerol and protein kinase $\mathrm{C}$ mitogen-activated protein kinase pathway functionality by interaction with Hog1p and Slt2p (Mpk1p). Eukaryot. Cell 2007, 6, 521-532.

93. Takabatake, R.; Ando, Y.; Seo, S.; Katou, S.; Tsuda, S.; Ohashi, Y.; Mitsuhara, I. MAP kinases function downstream of HSP90 and upstream of mitochondria in TMV resistance gene N-mediated hypersensitive cell death. Plant Cell Physiol. 2007, 48, 498-510.

94. Meiri, D.; Breiman, A. Arabidopsis ROF1 (FKBP62) modulates thermotolerance by interacting with HSP90.1 and affecting the accumulation of HsfA2-regulated sHSPs. Plant J. 2009, 59, 387-399.

95. Meiri, D.; Tazat, K.; Cohen-Peer, R.; Farchi-Pisanty, O.; Aviezer-Hagai, K.; Avni, A.; Breiman, A. Involvement of Arabidopsis ROF2 (FKBP65) in thermotolerance. Plant Mol. Biol. 2010, 72, 191-203.

96. Song, H.; Zhao, R.; Fan, P.; Wang, X.; Chen, X.; Li, Y. Overexpression of AtHsp90.2, AtHsp90.5 and AtHsp90.7 in Arabidopsis thaliana enhances plant sensitivity to salt and drought stresses. Planta 2009, 229, 955-964.

(C) 2012 by the authors; licensee MDPI, Basel, Switzerland. This article is an open access article distributed under the terms and conditions of the Creative Commons Attribution license (http://creativecommons.org/licenses/by/3.0/). 